\title{
ROBUST PREDICTIVE CONTROL BASED ON NEIGHBORING EXTREMALS
}

\author{
S. Gros, B. Srinivasan ${ }^{\dagger}$, and D. Bonvin \\ Laboratoire d'Automatique \\ École Polytechnique Fédérale de Lausanne \\ CH-1015 Lausanne, Switzerland
}

\begin{abstract}
The performance of a predictive controller is typically poor when the true plant evolution deviates significantly from that predicted by the model. A robust control approach that considers model uncertainty explicitly is then needed. However, it is often difficult to find a single input profile that works well for the range of uncertainty considered. Thus, multiple input profiles, i.e. one for each realization of the uncertainty, need to be determined. Unfortunately, this is computationally extremely expensive. This paper proposes an alternative approach that is based on neighboring extremals, where the multiple input profiles are computed using a simple feedback law, thereby reducing considerably the computational burden. The idea is illustrated via the simulation of a continuous stirred tank reactor and an inverted pendulum on a cart.
\end{abstract}

Keywords: Predictive control, Robust control, Optimal control, Neighboring extremals, Continuous stirred tank reactor, Inverted pendulum.

\section{INTRODUCTION}

The performance of a predictive controller lies in efficiently tackling problems with constraints and nonlinear dynamics, especially when analytical computation of the control law is difficult (Mayne et al., 2000; Scokaert and Mayne, 1998). Standard predictive control involves recalculating at every sampling instant the input ${ }^{2}$ that minimizes a criterion defined over a horizon window in the future, taking into account the current state of the process. Only the first part of the computed optimal input is applied to the process.

A key element in predictive control is the extensive use of the dynamic process model that, unfortunately, may not represent the reality ac-

\footnotetext{
$1 \dagger$ Present address :

Ecole Polytechnique de Montréal,

Génie Chimique, C.P. 6079,

Succ. Centre-Ville,

Montréal, Canada H3C3A7
}

curately. Thus, the predicted state evolution may differ from the future plant evolution. When the difference between the predicted and the true plant evolutions is significant (which occurs, for example, when the process is unstable), standard predictive control will be unable to provide the desired performance (Mayne et al., 2000; Rossiter et al., 1998). One solution to this problem consists of re-casting the problem into a robust framework, where optimization is performed by considering the uncertainty explicitly.

Standard robust predictive control computes an input that represent a compromise solution for the range of uncertainty considered (Bemporad and Morari, 1999; Lee and Yu, 1997; Kouvari-

\footnotetext{
2 The word input is considered here to be singular irrespective of whether there are one or several inputs. This choice is made in order to be able to distinguish between cases with a single or multiple input profiles corresponding to either a single nominal model or several models (one for each realization of the uncertainty).
} 
takis et al., 2000). Furthermore, to prove robust stability, it is important to guarantee that the final state is within some bounded set (Michalska and Mayne, 1993). When the dispersion of the open-loop predicted state is large, especially in the case of unstable processes, it may not be possible to find a feasible solution to the robust optimization problem. So, it becomes important to have a controlled state dispersion for which a time-varying state feedback has been used to maintain the states inside ellipsoidal feasible regions (Kouvaritakis et al., 2000).

However, due to the feedback introduced by the re-optimizations performed at subsequent sampling instants, the true state dispersion at the end of the prediction horizon will in fact be much smaller than the values given by open-loop prediction (Warren and Marlin, 2004). Hence, this inherent feedback needs to be incorporated in the robust predictive control formulation in order to reduce the conservatism and lead to feasible solutions.

There are two ways of expressing this inherent feedback in a robust optimization framework: (i) use multiple input profiles, i.e. one for each realization of the uncertainy, starting at the next sampling interval (Mayne et al., 2000; Scokaert and Mayne, 1998), and (ii) approximate the inherent feedback by a control law (Bemporad, 1998). The former is computationally expensive, while no generic method exist for the approximation in the latter. The present work proposes a novel way to use state feedback for MPC. It suggests using the neighboring extremal approach for approximating the feedback inherent to predictive control.

For small deviations away from the optimal solution, a linear approximation of the process dynamics and a quadratic approximation of the optimization cost are quite reasonable. In such a case, the theory of neighboring extremals (NE) provides a closed-form solution to the optimization problem (Bryson, 1999). Thus, the optimal input can be obtained using state feedback, which approximates the feedback provided by explicit numerical re-optimization.

The proposed NE-based approach can also be viewed as a novel way of performing robust optimization with multiple input profiles. Indeed, the scheme optimizes the multiple profiles, the nominal one exactly via explicit optimization and all the perturbed ones approximately via the NE approach. Since only one input profile is optimized explicitly, the computational complexity of the problem reduces considerably, while keeping the advantages of the robust optimization scheme with multiple input profiles. Note that the main emphasis is not in reducing the computational complexity of a general MPC problem as in (Wan and Kothare, 2003), but only in the context of robust predictive control.

The paper is organized as follows. Background material regarding predictive control and neighboring extremals is presented in Section 2. The standard robust predictive control scheme and robust predictive control with multiple input profiles are presented in Section 3. The robust predictive control based on the NE approach is proposed in Section 4. In Section 5, the various schemes are compared on two illustrative processes. Finally, concluding remarks are given in Section 6 .

\section{PRELIMINARIES}

\subsection{Standard Predictive Control}

Consider the nonlinear dynamic process:

$$
\dot{x}=F(x, u, \theta), \quad x(0)=x_{0}
$$

where the state $x$ and the input $u$ are vectors of dimension $n$ and $m$, respectively. $x_{0}$ represents the initial conditions, $\theta \in \Theta$ the vector of uncertain parameters that are assumed to lie in the admissible region $\Theta$, and $F$ the process dynamics.

In predictive control, the following optimization problem is solved repeatedly at discrete time instants:

$$
\begin{aligned}
& \min _{u\left(\left[t_{k}, t_{k}+T_{f}\right]\right)} J=\frac{1}{2} x\left(t_{k}+T_{f}\right)^{T} P x\left(t_{k}+T_{f}\right) \\
& +\frac{1}{2} \int_{t_{k}}^{t_{k}+T_{f}}\left(x(\tau)^{T} Q x(\tau)+u(\tau)^{T} R u(\tau)\right) d \tau \\
& \text { s.t. } \quad \dot{x}=F(x, u, \theta), \quad x\left(t_{k}\right)=x_{k} \\
& \quad x\left(t_{k}+T_{f}\right) \in \mathcal{X}
\end{aligned}
$$

where $P, Q$, and $R$ are positive-definite weighting matrices of appropriate dimensions, $\mathcal{X}$ the bounded region of state space where the final state should lie, $t_{k}$ the present time at which the optimization is performed, $T_{f}$ the prediction horizon, and $x_{k}$ the state measured or estimated at time $t_{k}$. The optimal input computed by solving (2) is represented by $u^{\star}\left(\left[t_{k}, t_{k}+T_{f}\right]\right)$. The importance of having a terminal cost, and also a bounded region for the final state for the sake of stability, is discused in (Mayne et al., 2000).

Let $h$ be the sampling period which, in general, is constant. The first part of the optimal input, $u^{\star}\left(\left[t_{k}, t_{k}+h\right]\right)$, is applied open loop, and the optimization problem is repeated at the time instant $t_{k+1}=t_{k}+h$. For implementation purposes, the infinite-dimensional input $u\left(\left[t_{k}, t_{k}+T_{f}\right]\right)$ is parameterized using a finite number of decision variables, typically a piecewise-constant approximation. 


\subsection{Neighboring Extremals}

Including the dynamic constraints of the optimization problem (2) in the cost function, the augmented cost function, $\bar{J}$, can be written as:

$$
\bar{J}=\Phi\left(x\left(t_{k}+T_{f}\right)\right)+\int_{t_{k}}^{t_{k}+T_{f}}\left(H-\lambda^{T} \dot{x}\right) d t
$$

where $\Phi(x)=\frac{1}{2} x^{T} P x, H=\frac{1}{2}\left(x^{T} Q x+u^{T} R u\right)+$ $\lambda^{T} F(x, u, \theta)$, and $\lambda(t) \neq 0$ is the $n$-dimensional vector of adjoint states or Lagrange multipliers for the system equations.

At the optimal solution, the first variation of $\bar{J}$ is given by (Bryson, 1999) (p. 64):

$$
\begin{aligned}
\Delta \bar{J} & =\left.\left(\Phi_{x}-\lambda^{T}\right) \Delta x\right|_{t_{k}+T_{f}} \\
& +\int_{t_{k}}^{t_{k}+T_{f}}\left[\left(H_{x}+\dot{\lambda}^{T}\right) \Delta x+H_{u} \Delta u\right] d \tau(4)
\end{aligned}
$$

where $\Delta x(t)=x(t)-x^{\star}(t)$ and $\Delta u(t)=u(t)-$ $u^{\star}(t)$, with $x^{*}$ and $u^{*}$ being the optimal state and input, respectively. The notation $a_{b}=\frac{\partial a}{\partial b}$ is used.

Upon convenient choice of the adjoint states, $\dot{\lambda}^{T}=$ $-H_{x}$ with $\lambda^{T}\left(t_{k}+T_{f}\right)=\Phi_{x}\left(t_{k}+T_{f}\right)$, the necessary conditions of optimality that are derived from $\Delta \bar{J}=0$ read:

$$
H_{u}=u^{T} R+\lambda^{T} F_{u}=0
$$

The second-order variation of $\bar{J}$ is given by (Bryson, 1999)(p. 317):

$$
\begin{gathered}
\Delta^{2} \bar{J}=\frac{1}{2} \Delta x\left(t_{k}+T_{f}\right)^{T} P \Delta x\left(t_{k}+T_{f}\right)+ \\
\frac{1}{2} \int_{t_{k}}^{t_{k}+T_{f}}\left[\begin{array}{ll}
\Delta x^{T} & \Delta u^{T}
\end{array}\right]\left[\begin{array}{ll}
H_{x x} & H_{x u} \\
H_{u x} & H_{u u}
\end{array}\right]\left[\begin{array}{c}
\Delta x \\
\Delta u
\end{array}\right] d \tau(6)
\end{gathered}
$$

Choosing $\Delta u$ to minimize $\Delta^{2} \bar{J}$ under the linear dynamic constraint

$$
\Delta \dot{x}=F_{x} \Delta x+F_{u} \Delta u
$$

represents a time-varying Linear Quadratic Regulator (LQR) problem, for which a closed-form solution is available:

$$
\begin{aligned}
& \Delta u(t)=-K(t) \Delta x(t) \\
& K=H_{u u}^{-1}\left(H_{u x}+F_{u}^{T} S\right) \\
& \dot{S}=-H_{x x}-S F_{x}-F_{x}^{T} S+H_{x u} K+S F_{u} K \\
& S\left(t_{k}+T_{f}\right)=P
\end{aligned}
$$

Controller (8), termed neighboring-extremal controller, will be used extensively in this paper.

\section{EXISTING APPROACHES TO ROBUST PREDICTIVE CONTROL}

\subsection{Standard Robust Predictive Control}

The state $x$ and thus also the cost function $J$ are functions of the vector of uncertain parameters $\theta$. For a given value of $\theta$, let denote by $x_{\theta}$ the state and $J_{\theta}$ the corresponding cost function. In robust predictive control, the uncertainty is handled as part of the optimization problem, which is solved repeatedly at discrete time instants:

$$
\begin{array}{rlr}
\min _{u\left(\left[t_{k}, t_{k}+T_{f}\right]\right)} \mathcal{E}\left(J_{\theta}, p(\theta)\right) & \\
\text { s.t. } & \dot{x}_{\theta}=F\left(x_{\theta}, u, \theta\right) & x_{\theta}\left(t_{k}\right)=x_{k} \\
& x_{\theta}\left(t_{k}+T_{f}\right) \in \mathcal{X}, \quad \forall \theta \in \Theta
\end{array}
$$

$$
\begin{aligned}
& J_{\theta}=\frac{1}{2} x_{\theta}\left(t_{k}+T_{f}\right)^{T} P x_{\theta}\left(t_{k}+T_{f}\right) \\
& +\frac{1}{2} \int_{t_{k}}^{t_{k}+T_{f}}\left(x_{\theta}(\tau)^{T} Q x_{\theta}(\tau)+u(\tau)^{T} R u(\tau)\right) d \tau
\end{aligned}
$$

where $\mathcal{E}\left(J_{\theta}, p(\theta)\right)$ is a general stochastic objective function that depends on the probability density function $p(\theta)$. A simple choice would be to use the expectation, but it has been noted in (Nagy and Braatz, 2003) that a robust control scheme based on expectation only leads to limited robustness improvement. To resolve this issue, terms related to the variance could be included.

The major difficulty with this formulation is that there may not be a solution, especially when the process is open-loop unstable. The state dispersion might be so large that it is not possible to find a single input $u\left(\left[t_{k}, t_{k}+T_{f}\right]\right)$ that satisfies $x_{\theta}\left(t_{k}+T_{f}\right) \in \mathcal{X}, \forall \theta \in \Theta$.

\subsection{Robust Predictive Control with Multiple Input Profiles}

Though computations in predictive control are performed open loop, there is inherent feedback due to state measurement/estimation and reoptimization. Since this feedback reduces the sensitivity to uncertainty, the state dispersion is often much smaller than what is predicted from an open-loop perspective.

The difficulty with the standard formulation (11) is that it does not take into account the fact that the optimization will be repeated at subsequent time instants. Hence, the idea of reformulating the robust predictive control problem and including re-optimization in the problem formulation (Mayne et al., 2000): 


$$
\begin{aligned}
& \min _{u_{\theta}\left(\left[t_{k}, t_{k}+T_{f}\right]\right)} \mathcal{E}\left(J_{\theta}, p(\theta)\right) \\
& \text { s.t. } \quad \dot{x}_{\theta}=F\left(x_{\theta}, u_{\theta}, \theta\right) \quad x_{\theta}\left(t_{k}\right)=x_{k} \\
& x_{\theta}\left(t_{k}+T_{f}\right) \in \mathcal{X}, \quad \forall \theta \in \Theta \\
& u_{\theta}(t)=\left\{\begin{array}{l}
u(t) \text { if } t_{k} \leq t<t_{k+1} \\
\bar{u}_{\theta}(t) \text { if } t_{k+1} \leq t \leq t_{k}+T_{f}
\end{array}\right. \\
& J_{\theta}=\frac{1}{2} x_{\theta}\left(t_{k}+T_{f}\right)^{T} P x_{\theta}\left(t_{k}+T_{f}\right) \\
& +\frac{1}{2} \int_{t_{k}}^{t_{k}+T_{f}}\left(x_{\theta}(\tau)^{T} Q x_{\theta}(\tau)+u_{\theta}(\tau)^{T} R u_{\theta}(\tau)\right) d \tau
\end{aligned}
$$

where $\bar{u}_{\theta}$ is the input computed for the uncertainty realization $\theta$. Problem (13) implies that (i) there is a single input profile that is independent of the realization of $\theta$ between $t_{k}$ and $t_{k+1}$, and (ii) several profiles corresponding to different realizations of $\theta$ between $t_{k+1}$ and $t_{k}+T_{f}$. This problem is computationally expensive to solve since $\bar{u}_{\theta}\left(\left[t_{k+1}, t_{k}+T_{f}\right]\right)$ needs to be determined for every realization of $\theta$. Note that, though $\bar{u}_{\theta}\left(\left[t_{k+1}, t_{k}+\right.\right.$ $\left.\left.T_{f}\right]\right)$ is important for calculating $u\left(\left[t_{k}, t_{k+1}\right]\right)$, it will never be implemented on the real process.

\section{ROBUST PREDICTIVE CONTROL BASED ON NEIGHBORING EXTREMALS}

In order to avoid computing the optimal input for many different realizations of $\theta$, a relationship between the uncertain parameters $\theta$ and the optimal input is needed. The idea proposed in this paper is to use the NE approach that provides the following relationship (see Subsection 2.2):

$$
\begin{aligned}
u_{\theta}\left(\left[t_{k+1}, t_{k}+T_{f}\right]\right) & =u_{\theta_{0}}\left(\left[t_{k+1}, t_{k}+T_{f}\right]\right) \\
& -K(t) \Delta x_{\theta}\left(\left[t_{k+1}, t_{k}+T_{f}\right]\right)(16)
\end{aligned}
$$

where $\theta_{0}$ is the nominal parameter vector and $\Delta x_{\theta}=x_{\theta}-x_{\theta_{0}}$. Optimization problem (13) then reduces to:

$$
\begin{aligned}
& \min _{u\left(\left[t_{k}, t_{k}+T_{f}\right]\right)} \mathcal{E}\left(J_{\theta}, p(\theta)\right) \\
& \text { s.t. } \quad \dot{x}_{\theta_{0}}=F\left(x_{\theta_{0}}, u, \theta_{0}\right), \quad x_{\theta_{0}}\left(t_{k}\right)=x_{k} \\
& \dot{x}_{\theta}=F\left(x_{\theta}, u_{\theta}, \theta\right), \quad x_{\theta}\left(t_{k}\right)=x_{k}, \quad \forall \theta \neq \theta_{0} \\
& x_{\theta}\left(t_{k}+T_{f}\right) \in \mathcal{X}, \quad \forall \theta \in \Theta \\
& u_{\theta}(t)= \begin{cases}u(t) & \text { if } t_{k} \leq t<t_{k+1} \\
u(t)-K\left(x_{\theta}-x_{\theta_{0}}\right) & \text { if } t_{k+1} \leq t \leq t_{k}+T_{f}\end{cases} \\
& J_{\theta}=\frac{1}{2} x_{\theta}\left(t_{k}+T_{f}\right)^{T} P x_{\theta}\left(t_{k}+T_{f}\right) \\
& +\frac{1}{2} \int_{t_{k}}^{t_{k}+T_{f}}\left(x_{\theta}(\tau)^{T} Q x_{\theta}(\tau)+u_{\theta}(\tau)^{T} R u_{\theta}(\tau)\right) d \tau
\end{aligned}
$$

Note that the decision variables in (17) are only $u\left(\left[t_{k}, t_{k}+T_{f}\right]\right)$ for the nominal plant and not $u_{\theta}\left(\left[t_{k}, t_{k}+T_{f}\right]\right)$ for all realizations. The input profiles for realizations other than the nominal one are computed using the NE controller.

The NE approach can be interpreted from two different viewpoints: (i) From the viewpoint of feedback, it is an approximation of the inherent feedback provided by the predictive control itself, and (ii) from the viewpoint of robust predictive control with multiple input profiles, the NE approach computes a first-order approximation of the optimal input profiles that correspond to the various realizations of $\theta$. Note that, as in Subsection 3.2, the input computed via the NE approach will never be implemented on the real process since it is computed for the time interval $\left[t_{k+1}, t_{k}+T_{f}\right]$. Thus, the NE feedback is a fictitious one that serves only a computational purpose. Since the proposed NE approach is only an approximation of robust control with multiple input profiles, it should exhibit inferior performance. However, in many examples that have been worked out, the NE approach led to slightly better performance then robust control with multiple input profiles. This can be attributed to the fact that robust control with multiple input profiles requires the solution of an optimization problem with a large number of decision variables and a lack of sensitivity of the objective function. Hence, it might be advantageous to use a well-posed feedback law instead of an ill-posed open-loop problem.

Though this paper presents no stability proof for the proposed NE-based robust predictive controller, one can forsee steps pointing to robust stability: (i) Stability of robust predictive control with multiple input profiles (Mayne et al., 2000), (ii) proof that NE-based robust predictive control is a first-order approximation to that with multiple input profiles (see preliminary work in this direction by (Ronco et al., 2001)), and (iii) effect of the approximation error on stability.

The computation of the feedback law $K(t)$ adds some load to the optimization algorithm. It requires the computation of the adjoints $\lambda(t)$ and the sweep matrix $S(t)$. These computations are performed using backward integration based on the optimal input found for the nominal system. Integration of the adjoints can require some precaution as these variables can be unstable. However, experience has shown that an appropriate choice of the cost function and of the prediction horizon can ease the integration significantly (e.g. the weights in the cost function and the prediction horizon are chosen so that the state predictions converge to the reference values within the prediction horizon). Computing the optimal input for the nominal system and integrating the adjoints and sweep matrix is considerably less demanding than computing the optimal input for several models in the model set. 


\section{ILLUSTRATIVE EXAMPLES}

Two examples will be presented in this section. The first one is a continuous stirred tank reactor (CSTR), for which standard predictive control stabilizes the process but gives poor performance. However, standard robust predictive control is unstable, while the proposed methodology gives good performance. The second example considers an inverted pendulum, for which robust control with multiple input profiles is required for stability. Also, since the second example is numerically less time consuming, dispersion plots are included to illustrate the effect of uncertainty on control performance.

\subsection{Control of a Continuous Stirred Tank Reactor}

5.1.1. Process Model: Concentration control in a CSTR with constant cooling jacket temperature is considered. There is a single exothermic chemical reaction, $A \rightarrow B$, and the manipulated variable is the inlet feed rate. The model equations are:

$$
\begin{aligned}
\dot{c}_{A}= & -k_{0} c_{A} e^{\frac{-E}{R T}}+\frac{F}{V}\left(c_{A_{i n}}-c_{A}\right) \\
\dot{T}= & \frac{(-\Delta H)}{\rho c_{p}} k_{0} c_{A} e^{\frac{-E}{R T}}+ \\
& \frac{F}{V}\left(T_{i n}-T\right)+\frac{U A}{V \rho c_{p}}\left(T_{c}-T\right)
\end{aligned}
$$

where $c_{A}$ is the concentration of species $A, T$ the reactor temperature, $T_{i n}$ the inlet temperature, $T_{c}$ the cooling jacket temperature, $F$ the feed rate of A, $k_{0}$ the pre-exponential factor, $E$ the activation energy, $R$ the gas constant, $V$ the reactor volume, $\Delta H$ the reaction enthalpy, $\rho$ the density, $c_{p}$ the heat capacity, $U$ the heat transfer coefficient, and $A$ the heat transfer area. The parameter values, taken from (Eker and Nikolaou, 2002), are listed in Table 1.

The uncertainty regards the pre-exponential factor $k_{0}$ that can take any value in the range [ $5.66 \times$ $\left.10^{7}, 8.5 \times 10^{7}\right]$ with equal probability. The value used for simulating the reality is $k_{0}^{\text {real }}=8.14 \times$ $10^{7}[1 / h]$, which is larger than the nominal model value $k_{0}^{\text {nom }}=7.08 \times 10^{7}[1 / h]$, i.e. the reality is more reactive than predicted by the model.

As shown in Figure 1, for a feed rate $F=5\left[\mathrm{~m}^{3} / h\right]$, the model exhibits three equilibrium points, $A$, $B$ and $C$, that correspond to different values of $c_{A}$ and $T$. The equilibrium points $A$ and $C$ are stable, while the equilibrium point $B$ is unstable. The control objective is to drive the process from the stable equilibrium point $A$ to the unstable equilibrium point $B$ that is noted $\left(c_{A, r e f}, T_{r e f}\right)$.
The numerical values for the points $A$ and $B$ are displayed in Table 2.

\begin{tabular}{|l|l|l|}
\hline$V$ & 1.36 & $\mathrm{~m}^{3}$ \\
$c_{A, \text { in }}$ & 8008 & $\mathrm{~mol} / \mathrm{m}^{3}$ \\
$k_{0}^{\text {nom }}$ & $7.08 \times 10^{7}$ & $1 / \mathrm{h}$ \\
$E / R$ & 8375 & $\mathrm{~K}$ \\
$T_{\text {in }}$ & 373.3 & $\mathrm{~K}$ \\
$T_{c}$ & 532.6 & $\mathrm{~K}$ \\
$\rho$ & 800.8 & $\mathrm{~kg} / \mathrm{m}^{3}$ \\
$c_{p}$ & 3140 & $\mathrm{~J} /(\mathrm{kgK})$ \\
$U A$ & $7.04 \times 10^{6}$ & $\mathrm{~J} /(\mathrm{hK})$ \\
$(-\Delta H)$ & 69775 & $\mathrm{~J} / \mathrm{mol}$ \\
\hline
\end{tabular}

\begin{tabular}{|l|l|l|}
\hline & $c_{A}\left[\mathrm{~mol} / \mathrm{m}^{3}\right]$ & $T[K]$ \\
\hline Equ. point A & 1586.08 & 544.72 \\
\hline Equ. point B & 4786.23 & 487.79 \\
\hline
\end{tabular}

Table 2. Equilibrium points $A$ and $B$ for the model corresponding to $F=$ $5\left[m^{3} / h\right]$

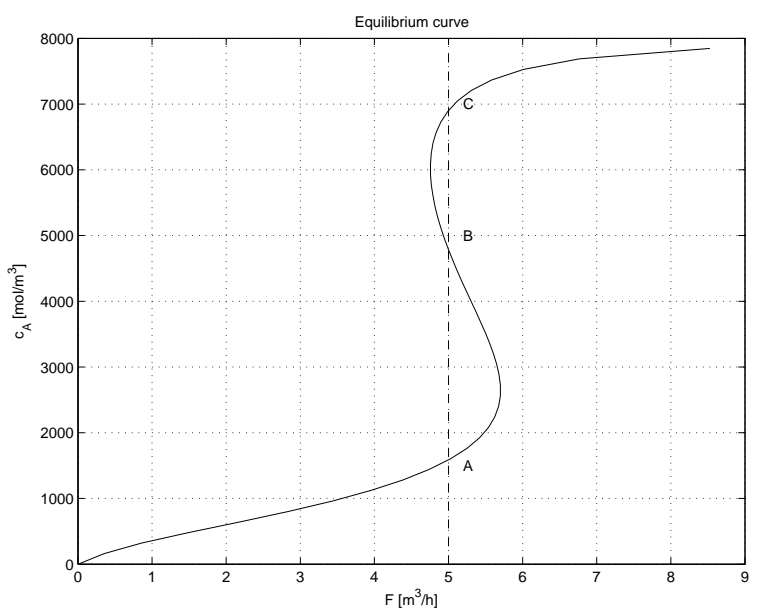

Fig. 1. Values of $c_{A}$ at equilibrium as a function of the feed rate

5.1.2. Integral Control: The equilibrium point varies with $k_{0}$, i.e. $F=5\left[\mathrm{~m}^{3} / \mathrm{h}\right]$ no longer corresponds to the equilibrium points $A$ and $B$ for the real process. Hence, any proportional controller will seek a compromise between meeting $F=5\left[m^{3} / h\right]$ and $c_{A}=c_{A, r e f}$ at steady state. Fortunately, the resulting steady-state error can be eliminated via integral control. For this, an additional state $I$ with the following dynamics is included:

$$
\dot{I}(t)=c_{A, \text { ref }}-c_{A}(t) \quad I(0)=0
$$

5.1.3. Control Parameters: All control predictive schemes investigated below share the following features: (i) re-optimization of the input at each sampling instant, with $h=0.2[h]$, (ii) prediction horizon $T_{f}=1[h]$, (iii) control horizon of one sampling period, i.e. the control sequence for $t_{k} \leq t \leq t_{k}+h$ is applied, leaving the rest of the 
sequence unused, (iv) piecewise-constant parameterization of the input $F(t)$ with time intervals of length $h$, and (iv)

$P=\left[\begin{array}{lll}1 & 0 & 0 \\ 0 & 0 & 0 \\ 0 & 0 & 4\end{array}\right], Q=\left[\begin{array}{lll}1 & 0 & 0 \\ 0 & 0 & 0 \\ 0 & 0 & 0\end{array}\right]$ and $R=10^{3}$.

Note that the temperature is not pushed to its reference, as only concentration control is considered. The stochastic objective function chosen for this example is the expectation of $J_{k_{0}}$. The probability density of the uncertain parameter $k_{0}$ is supposed uniform. The objective function is based on the expectation and is approximated as $\frac{1}{3} \sum_{j=1}^{3} J_{k_{0}^{j}}$, with $k_{0}^{1}=5.66 \times 10^{7}[1 / h], k_{0}^{2}=$ $7.08 \times 10^{7}[1 / h]$ and $k_{0}^{3}=8.5 \times 10^{7}[1 / h]$. As noted before though this choice leads to limited robustness improvement, it is justified by the fact that it is quite standard approach in the literature.

5.1.4. Standard Predictive Control: The standard non-robust re-optimization scheme (2) is applied first. The control is computed from a single nominal model with $k_{0}^{n o m}=7.08 \times 10^{7}[1 / h]$ and applied to the simulated reality with $k_{0}^{\text {real }}=$ $8.14 \times 10^{7}[1 / h]$. Simulation results are displayed in Figure 2, which shows that, if this scheme ultimately converges, it applies unnecessary large (of the bang-bang type) control to the process. This in turn causes large variations in $c_{A}$ which in fact goes to zero for a short time (complete consumption of $A$ due to zero feed and unacceptably-high temperatures). Performance improvement clearly calls for a robust control approach.
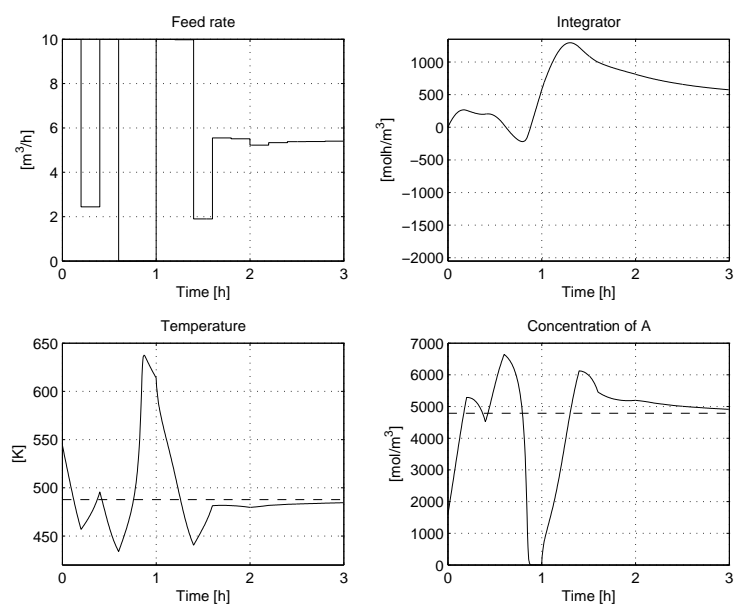

Fig. 2. Standard predictive control of CSTR

5.1.5. Standard Robust Predictive Control: The robust predictive control scheme considered here uses three models. Optimization scheme (11) computes a single input profile for all three models. The simulation results are displayed in Figure 3. They show that this approach does not converge.
The optimization is unable to find a single input profile that works well with all three models. Hence, the predicted value of the cost function is high and does not decrease with the number of re-optimizations. No final state constraint can be imposed since this optimization is unable to provide a feasible solution.
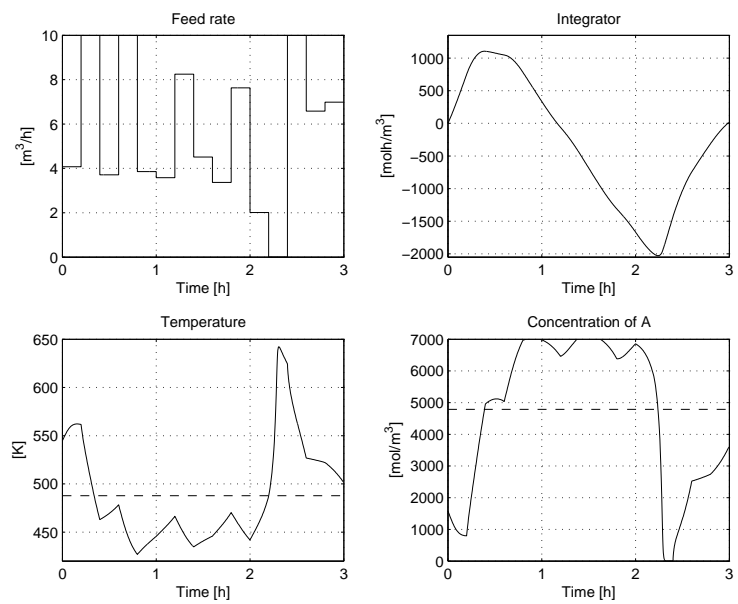

Fig. 3. Standard robust predictive control of CSTR

5.1.6. Robust Control with Multiple Input Profiles: The robust control scheme with multiple input profiles (13) is studied next. The same three models are used but, at each re-optimization, three different control sequences are computed, i.e. one for each model. The input for the first sampling interval is the same for all three models, but distinct for each model thereafter. The results of the simulation are displayed in Figure 4. The computational burden is very high, the average duration of one re-optimization is approximately $1.21[h]$. Also, since the number of decision variables and the number of simulations to be performed increase with the number of realizations considered, the computational time increases quadratically with the number of uncertainty realizations.

It can be noted that the convergence is not perfect since there remain oscillations in the input and states. This is due to the numerical difficulties and the sensitivity issues associated with this scheme. Indeed, since the optimization is characterized by a large number of variables and thus a lack of sensitivity of the cost function, the numerical optimization algorithm struggles to find the optimum and stops at sub-optimal values.

\subsubsection{Robust Control based on Neighboring Ex-} tremals: At the re-optimization at time $t_{k}$, an optimal input is computed for the nominal model using a piecewise-constant input parameterization of length $h$. Based on this input, a NE controller 

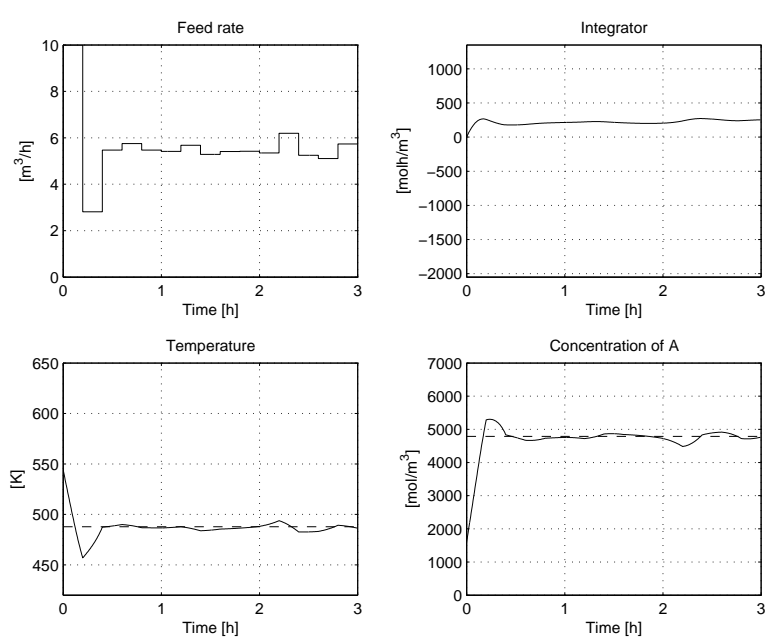

Fig. 4. Robust control of CSTR with multiple input profiles

is designed to generate $u\left[t_{k}+h, t_{k}+T_{f}\right]$ for each of the three models. A new NE controller is therefore computed at each re-optimization. Simulation results are displayed in Figure 5 and show that this approach works well on this process. The computational burden is much lower than with multiple input profiles: The average duration of a re-optimization is of the order of $0.1[h]$, a 12 fold reduction compared to scheme (13), making real-time implementation realistic since the reoptimization time can be kept smaller than the sampling period $h$. Moreover, with the NE approach, the computational time increases linearly with the number of models used to represent the uncertainty.
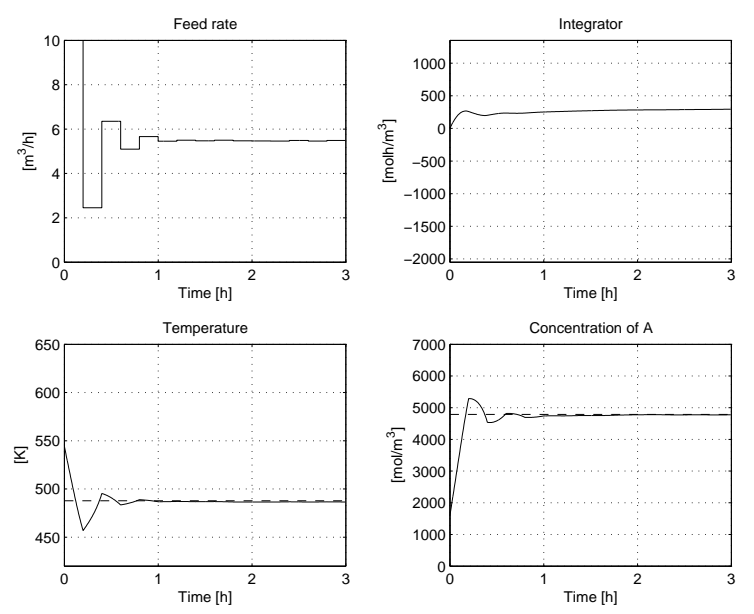

Fig. 5. Robust control of CSTR with neighboring extremals

\subsection{Control of an Inverted Pendulum}

5.2.1. Process Model: This section illustrates the application of both standard and robust predictive control schemes to an inverted pendulum on a cart. Ignoring the cart dynamics for simplicity, the model equations read (Ronco et al., 2001):

$$
\begin{aligned}
& \dot{x}_{1}=x_{2} \\
& \dot{x}_{2}=\frac{m l}{J}\left[\sin \left(x_{1}\right) g-\cos \left(x_{1}\right) u\right]
\end{aligned}
$$

where $x_{1}$ is the pendulum angle, $x_{2}$ its rotational velocity, and $u$ the control acceleration. The control objective consists of regulating the pendulum around the upright position, starting from the downward position $x_{0}=\left[\begin{array}{ll}\pi & 0\end{array}\right]$. The following numerical values are used: $m=1[\mathrm{~kg}], g=9.81\left[\frac{\mathrm{m}}{\mathrm{s}^{2}}\right]$, $l=1[\mathrm{~m}]$ and $J=1\left[\mathrm{~kg} \cdot \mathrm{m}^{2}\right]$. In addition, the control is constrained, $-5\left[\frac{\mathrm{m}}{\mathrm{s}^{2}}\right] \leq u \leq 5\left[\frac{\mathrm{m}}{\mathrm{s}^{2}}\right]$. For all techniques, the same sampling period $h=0.2[s]$ is used.

In the following simulations, it is assumed that the mass of the pendulum is unknown but lies somewhere between $0.5[\mathrm{~kg}]$ and $1.5[\mathrm{~kg}]$ with equal probability. As in the first example, the stochastic objective function is chosen as the expectation of $J_{m}$. The probability density of the uncertain parameter $m$ is considered uniform. The expectation is approximated as $\frac{1}{3} \sum_{j=1}^{3} J_{m_{j}}$, with $m_{1}=$ $0.5[\mathrm{~kg}], m_{2}=1[\mathrm{~kg}]$ and $m_{3}=1.5[\mathrm{~kg}]$. The predictive control schemes are applied to a simulated reality with a mass $m^{\text {real }}=1.32[\mathrm{~kg}]$, thus making the real process slower than the nominal model $\left(m^{\text {nom }}=1[k g]\right)$.

5.2.2. Control Parameters: All schemes share the following features: (i) re-optimization of the input at each sampling instant, (ii) prediction horizon $T_{f}=1 s$, (iii) control horizon of one sampling period, (iv) the condition $x\left(t_{k}+T_{f}\right) \in \mathcal{X}$ is not enforced, (v) $P=10 I, Q=10^{-2}, R=0.02$, where $I$ is the identity matrix, and (vi) the input parameterization considers $u(t)$ constant over the time interval $\left[t_{k}, t_{k}+h\right]$; the rest of the input trajectory is obtained using the shooting method (Lewis, 1986), with the initial conditions of the adjoint variables serving as parameters.

5.2.3. Simulation results: The standard predictive control scheme (2) is applied first (Figure 6). The approach does not work since the sampling period is too large for the control scheme to converge.

The standard robust predictive control scheme (11) that computes a single input profile for all three models is considered (Figure 6). This approach does not work either since it is unable to find a single input profile that works well with all three models.

The robust control scheme with multiple input profiles (13) is studied next (Figure 7). The computational burden is very high, the duration of one re-optimization (when the process is not close to the reference) is approximately $1.44[h]$. The convergence is quite good. 

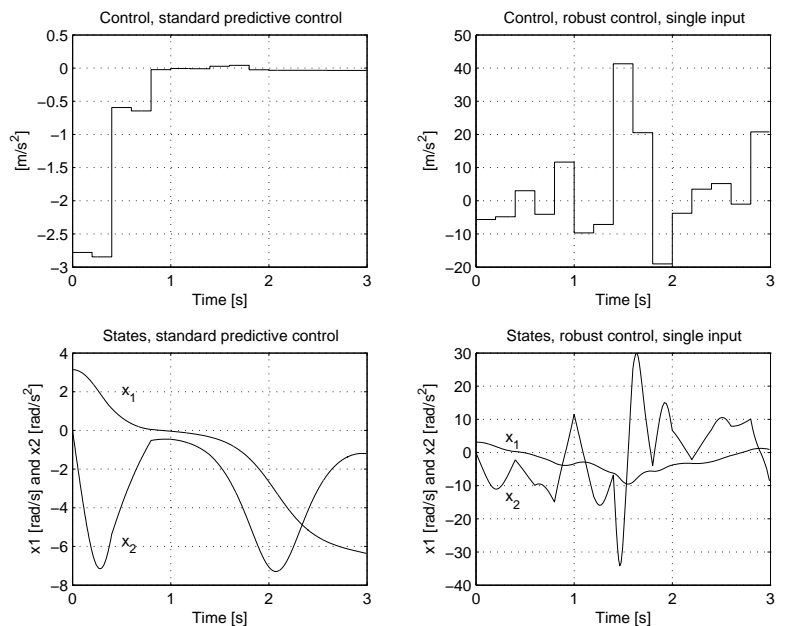

Fig. 6. Standard and robust predictive control of inverted pendulum

Finally, the proposed NE-based approach (17) is used. At each re-optimization, an optimal input is computed for the nominal model using the shooting method. Based on this optimal input, a NE controller is designed to generate the optimal trajectory for each of the three models in the interval $\left[t_{k}+h, t_{k}+T_{f}\right]$. Simulation results are displayed in Figure 7 and show that this approach works well. The computational burden is much lower than with multiple input profiles, the duration of a re-optimization (when the process is not close to the reference point) being of the order of 12 minutes.
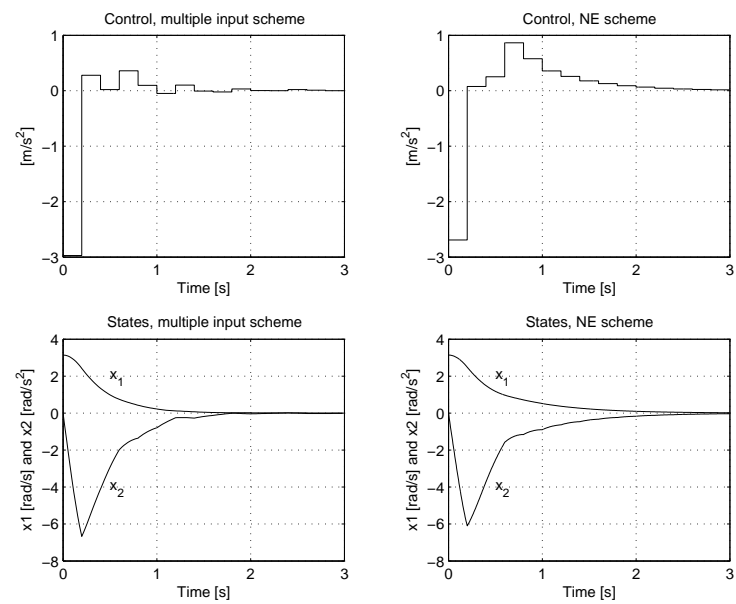

Fig. 7. Robust control of inverted pendulum with multiple input profiles (left) and NE controller (right)

5.2.4. Dispersion plots: Figure 8 (left) illustrates the predictive feature of standard predictive controllers, whereby the input computed in the first iteration is applied to both model and reality for the prediction horizon of 1 second. The deviations in state trajectories between model and reality are quite significant, especially for the velocity $x_{2}$. For example, the model predicts zero position and velocity after one second, whereas the reality is far from that. This is the main reason for the failure of standard predictive control.
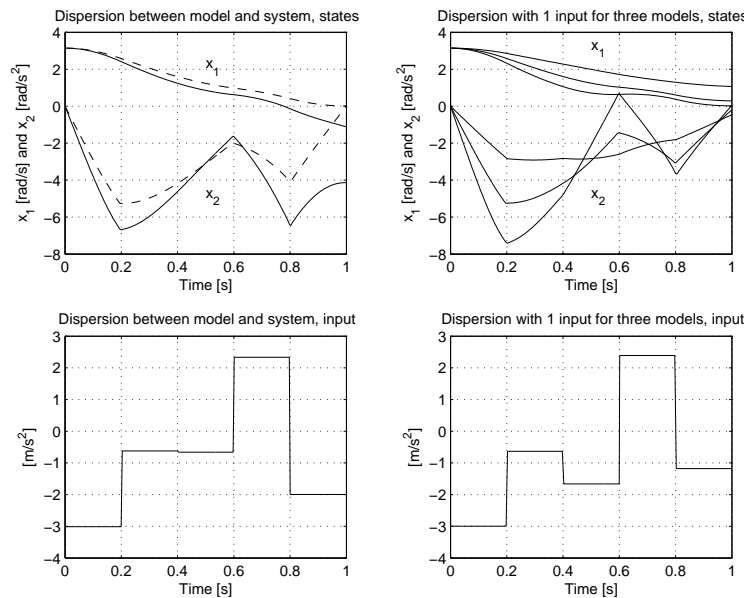

Fig. 8. Deviation between model (dashed line) and simulated process (plain line) for standard predictive control (left column) for a given input; dispersion of the three models (right column) for a given input

In standard robust predictive control, the input computed in the first iteration can bring the position and velocity in all three models near but not exactly at zero (Figure 8, right). However, since the process is unstable, this residual dispersion is still sufficient to make the closed-loop system unstable.
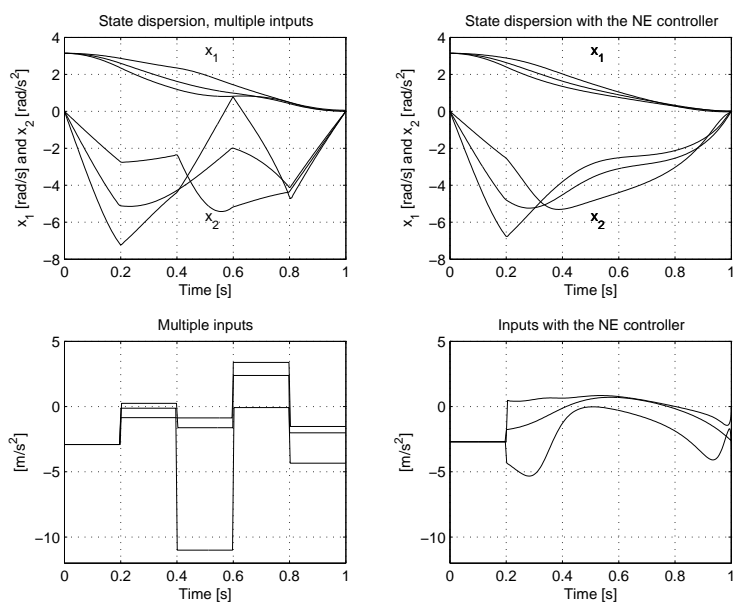

Fig. 9. Dispersion of the three models with multiple inputs profiles (left) and NE controller (right)

Figure 9 shows the same dispersion for the robust predictive controller with multiple input profiles (left) and the NE controller (right). While the first part of the input is unique for all models, the rest of the sequence differs for each model. These input parts are either optimized or generated by the NE controller. Both the optimized multiple input profile approach and the NE controller are able to drive the three model predictions very 
close to zero at the end of the prediction horizon. This explains the success of these methods in an uncertain scenario. It is interesting to note that the variations within the interval is larger for the optimized multiple profile approach than for the NE controller.

\section{CONCLUSION}

This paper has addressed the problem of robust predictive control of processes for which the openloop prediction of the future state evolution leads to very conservative results. The fact that there is feedback via re-optimization needs to be incorporated in the state prediction. This is done in this paper using the NE approach that is compared to other approaches on simple chemical and mechanical examples.

Stability and performance of the NE-based robust predictive control scheme have not been addressed in this paper. These issues, in particular the validity of the first-order approximation and the effect of the approximation on stability, will form the subject of future research. The feedback computed using the NE approach is only used as a fictitious input for computational purposes. Yet, its use for direct implementation is another promising research direction.

\section{REFERENCES}

Bemporad, A. (1998). Reducing conservatism in predictive control of constrained systems with disturbances. In: 37th IEEE Control and Decision Conference. Tampa, FL. pp. 1384-89.

Bemporad, A. and M. Morari (1999). Robust Model Predictive Control: a Survey. Springer Verlag.

Bryson, A. E. (1999). Dynamic Optimization. Addison-Wesley, Menlo Park, California.

Eker, S. A. and M. Nikolaou (2002). Linear control of nonlinear systems: Interplay between nonlinearity and feedback. AIChE J. 48(6), 1957-1980.

Kouvaritakis, B., J. A. Rossiter and J. Schuurmans (2000). Efficient robust predictive control. IEEE Trans. Automat. Contr. 45(8), 1545-49.

Lee, J. H. and Z. Yu (1997). Worst-case formulations of model-predictive control for systems with bounded parameters. Automatica 33(5), 763-781.

Lewis, F. L. (1986). Optimal Control. John Wiley, New York.

Mayne, D. Q., J. B. Rawlings, C. V Rao and P. O. M. Scokaert (2000). Constrained model predictive control: Stability and optimality. Automatica 36(6), 789-814.
Michalska, H. and D. Q. Mayne (1993). Robust receding horizon control of constrained nonlinear systems. IEEE Trans. Automat. Contr. 38(11), 1623-33.

Nagy, Z. K. and R. D. Braatz (2003). Robust nonlinear model predictive control of batch processes. AIChE Journal 49(7), 1776-1786.

Ronco, E., B. Srinivasan, J. Y. Favez and D. Bonvin (2001). Predictive control with added feedback for fast nonlinear systems. In: European Control Conference. Porto, Portugal. pp. 3167-3172.

Rossiter, J. A., B. Kouvaritakis and M. J. Rice (1998). A numerically robust state-space approach to stable-predictive control strategies. Automatica 34(1), 65-73.

Scokaert, P. O. and D. Q. Mayne (1998). Minmax feedback model predictive control for constrained linear systems. IEEE Trans. Automat. Contr. 43, 1136-1142.

Wan, Z. and M. V. Kothare (2003). An efficient off-line formulation of robust model predictive control using linear matrix inequalities. Automatica 39, 837-846.

Warren, A. L. and T. E. Marlin (2004). onstrained mpc under closed-loop. Proceedings of the American Control Conference 5, 4607-4612. 